\title{
Creating and implementing objective structured clinical exams for use in pediatric nursing education in Ghana: Reflections and lessons learned
}

\author{
Barbara Couper*1, Stephanie de Young ${ }^{1}$, Mary Douglas $^{1}$, Sawdah Esaka Aryee ${ }^{2}$, Roxana Salehi ${ }^{1}$, Charity Djokoto $^{2}$, \\ Augustine Asamoah ${ }^{2}$ \\ ${ }^{1}$ Centre for Global Child Health, Hospital for Sick Children, Canada \\ ${ }^{2}$ Ghana College of Nurses and Midwives, Ghana
}

Received: December 15, 2021

DOI: $10.5430 /$ jnep.v12n6p9

\author{
Accepted: January 12, 2022 \\ Online Published: January 24, 2022 \\ URL: https://doi.org/10.5430/jnep.v12n6p9
}

\begin{abstract}
The use of the Objective Structured Clinical Exam (OSCE) to measure the clinical competency of health care professionals is well established in many training institutions globally because of their high validity and reliability. In the context of a pediatric nursing partnership program in Ghana (the SickKids-Ghana Pediatric Nursing Education Partnership [PNEP]), we aimed to develop a national, accredited, competency-based curriculum. The integration of the OSCE into the curriculum was novel in the Ghanaian setting. It served as a standardized method for student assessment of higher clinical competencies, including both skills and attitudes, compared to conventional methods. Using mixed-methods, we previously found that the PNEP curriculum effectively increased graduates' knowledge, confidence, and clinical skill in pediatric nursing. This manuscript aims to reflect on the development of the OSCE stations, case scenarios, and rating materials, with particular reference to the methodological approach and lessons learned. We further reflect on the feedback from faculty and students pertaining to the usefulness of the OSCE as an assessment tool and the inclusion of standardized patients to assess communication skills. Adaptations required to safely conduct the OSCEs in the context of the COVID-19 pandemic have also been highlighted. Our findings throughout the OSCE development, implementation, and testing processes in Ghana could aid and inform similar tool development for nursing education institutions in the West African region and other resource-limited settings.
\end{abstract}

Key Words: OSCE, Nursing education, Paediatric nursing

\section{BACKGROUND}

Within healthcare and education settings, interactive, competency-based examinations can offer a more holistic approach to the evaluation of clinical skills and performance. ${ }^{[1,2]}$ The Objective Structured Clinical Exam (OSCE) is commonly used to assess healthcare providers and involves a set of timed assessment stations. At each station, students are presented with a case scenario based on a real-life clinical situation and required to actively demonstrate how to man- age the fixed case. Correspondingly, assessors are trained to evaluate students' performance according to a standardized rating tool and techniques. Compared to a conventional written or oral examination, which tests knowledge recall, incorporating OSCEs allows for appraisal of actual clinical skills and attitudes, which are considered a higher level of competency. ${ }^{[3]}$

Designed to simulate a clinical environment, OSCEs typically take place in a lab or classroom and incorporate the use

\footnotetext{
* Correspondence: Barbara Couper; Email: barbara.couper@sickkids.ca; Address: Centre for Global Child Health, Hospital for Sick Children, Canada.
} 
of mannequins or simulation models. Standardized patients (SPs) recruited from the community and trained to portray a patient or family member can also be built-in. ${ }^{[4,5]}$ OSCEs offer each student a similar experience, and ensure the same level of difficulty is presented by controlling for confounding variables that might otherwise present in a clinical setting and affect standardization (e.g. an uncooperative patient). They have been shown to allow for better and more reliable competency assessment compared with exams on real patients. ${ }^{[6,7]}$ Furthermore, acute and complex cases that may not be appropriate or available for testing in a clinical setting, such as emergencies or deteriorating patients, can be integrated. ${ }^{[8]}$

Conversely, a limitation of the OSCE includes variability in preparation and training of OSCE assessors (called raters), which influences exam validity. ${ }^{[9]}$ Rater bias has also been noted as a potential factor resulting in inequalities during assessments, either through the assignment of unequal rating scores or providing differing prompts to students. ${ }^{[10,11]}$ Providing thorough and effective training can substantially reduce rater error. ${ }^{[12]}$

As part of nursing education, use of the OSCEs has been described in diverse settings as an important evaluation tool by nurse educators. ${ }^{[7]}$ Most experiences are reported in highincome country settings, as opposed to the resource-limited settings experienced in lower- and middle-income countries. There are, however, emerging examples of the implementation of formal OSCEs in Central and East Africa in the literature. ${ }^{[1,13-15]}$ In the West African region, there has also been momentum to integrate OSCEs in health professional education. ${ }^{[16]}$

In Ghana, nursing assessments have traditionally employed practical exams, meaning exams are conducted on real patients in a clinical setting. In the context of a pediatric nursing partnership program in Ghana, we aimed to develop a national, accredited, competency-based curriculum. The integration of the OSCE into the curriculum served as a novel, standardized method for student assessment of higher competencies. This manuscript aims to reflect on the development of the OSCE, with particular reference to the methods used and lessons learned. Details on the effectiveness of the curriculum and the use of OSCEs for assessing clinical skills to evaluate program effectiveness have been published elsewhere. $^{[17]}$

\section{MethodS}

\subsection{The pediatric nursing education partnership}

In 2015, the Pediatric Nursing Education Partnership (PNEP) was developed to generate a specialized pediatric nursing curriculum in Ghana and provide experienced registered nurses 10
(RNs) with further competency-based education and certification in pediatric clinical care. The five-year partnership was a collaboration between the Hospital for Sick Children (SickKids), a pediatric hospital in Toronto, Canada, and the Ghana College of Nurses and Midwives (GCNM), an agency of the Ghana Ministry of Health based in Accra, Ghana, responsible for postgraduate nursing education and the local implementing partner. RNs admitted to the program were required to be currently licensed by the Nursing and Midwifery Council of Ghana (NMC); hold a diploma in nursing (at minimum); and have at least 3 years of work experience. Across 3 teaching sites (Accra, Kumasi, and Tamale), PNEP trained 501 certified pediatric RNs via a 1-year postgraduate program, which included 11 cohorts. The curriculum was found to effectively increase graduates' knowledge, confidence, and clinical skill in pediatric nursing. ${ }^{[17]}$ PNEP formally completed in September 2020, although the curriculum continues to be used by GCNM to train pediatric RNs.

\subsection{Roles}

PNEP focused on a competency-based curriculum, therefore we developed an OSCE specific to the program's foundational skills and the Ghanaian clinical context. There were many individuals involved in the development and rollout (see Table 1). Those at the stakeholder level developed the OSCE materials and provided ongoing oversight, while the examination team was responsible for carrying out the student assessments.

\subsection{OSCE content development and structure}

At the content development stage, OSCE core principles and best practices were reviewed to identify the key skills to test. ${ }^{[18,19]}$ Three stations, physical assessment, emergency management, and communication, were developed for each OSCE, reflecting a range of clinical competencies and practical skills foundational to pediatric nursing practice (Table 2). Each station was designed to take a maximum of 10 minutes.

Case scenario development was a collaborative process between SickKids and GCNM to ensure they were comprehensive, culturally relevant, and relatable to the Ghanaian clinical environment. A bank of cases was developed for each station, which allowed for the assessment of different cases with each group of students. Protocols were developed to ensure standardization between teaching sites and at each administration point. These described the procedures and OSCE tools; facilitated consistent communication with the students; and advised as to how to deal with any challenges that arose (e.g. student failure or emergency). The OSCEs were used at multiple timepoints throughout PNEP, serving to assess both students' clinical performance and as a program evaluation measure (see Table 3 ).

ISSN 1925-4040 E-ISSN 1925-4059 
Table 1. Roles within the OSCE development and rollout for PNEP

\begin{tabular}{|c|c|}
\hline Roles & Description \\
\hline \multicolumn{2}{|l|}{ Stakeholders } \\
\hline SickKids & $\begin{array}{l}\text { - Included nurse educators and evaluation specialist } \\
\text { - Collaborated to develop OSCE content, materials, and procedures } \\
\text { - Provided mentorship and training for the OSCEs }\end{array}$ \\
\hline GCNM & $\begin{array}{l}\text { - Included faculty, clinical instructors, preceptors, and evaluation staff } \\
\text { - Collaborated to develop OSCE content, materials, and procedures } \\
\text { - Made up the examination team (below) }\end{array}$ \\
\hline NMC & $\begin{array}{l}\text { - Deputy Registrar provided oversight at initial OSCE to exchange best practices and ensure } \\
\text { standards were met for certification }\end{array}$ \\
\hline \multicolumn{2}{|l|}{ Examination team } \\
\hline Raters & - Evaluated student performance on the case scenarios \\
\hline Station coordinators & $\begin{array}{l}\text { - Supported the rater during the exam by resetting stations between students, holding up any } \\
\text { cards with findings (i.e. vital signs, lab values) for the student, and assisting if there were } \\
\text { problems with the simulation supplies }\end{array}$ \\
\hline Lead coordinators & $\begin{array}{l}\text { - Led the OSCE process at each site } \\
\text { - Communicated with students before they entered the assessment } \\
\text { - Coordinated a repeat assessment if a student failed }\end{array}$ \\
\hline Standardized patients & $\begin{array}{l}\text { - Community members selected by GCNM } \\
\text { - Portrayed parent of a sick child } \\
\text { - Contributed to the evaluation of student performance in the communication station }\end{array}$ \\
\hline \multicolumn{2}{|l|}{ Auxiliary personnel } \\
\hline OSCE educators & - Led the training sessions for the raters and SPs \\
\hline Monitoring and evaluation staff & $\begin{array}{l}\text { - GCNM staff } \\
\text { - Managed data collection, storing, transporting confidential materials, data entry and analysis }\end{array}$ \\
\hline
\end{tabular}

Table 2. Components of the OSCE developed for PNEP in Ghana

\begin{tabular}{|c|c|c|c|}
\hline \multirow{2}{*}{ Characteristics } & \multicolumn{3}{|l|}{ Stations } \\
\hline & Physical assessment & Emergency management & Communication \\
\hline $\begin{array}{l}\text { Focus of case } \\
\text { scenarios }\end{array}$ & $\begin{array}{l}\text { Assessment of a single body system (i.e. } \\
\text { respiratory system) and identification of } \\
\text { abnormal finding using a } \\
\text { developmentally appropriate approach }\end{array}$ & $\begin{array}{l}\text { Assessment of a pediatric } \\
\text { emergency and management } \\
\text { of Airway, Breathing, } \\
\text { Circulation and/or } \\
\text { Dehydration }\end{array}$ & $\begin{array}{l}\text { Navigate a challenging } \\
\text { discussion with a caregiver of a } \\
\text { child requiring hospital care } \\
\text { through effective } \\
\text { communication and } \\
\text { family-centered principles }\end{array}$ \\
\hline \multirow{2}{*}{$\begin{array}{l}\text { Personnel } \\
\text { involved }\end{array}$} & Rater & Rater & Rater \\
\hline & Station coordinator & Station coordinator & Standardized patient \\
\hline \multirow{3}{*}{$\begin{array}{l}\text { Documents } \\
\text { required }\end{array}$} & Rater and station coordinator directions & $\begin{array}{l}\text { Rater and station coordinator } \\
\text { directions }\end{array}$ & Rater directions \\
\hline & $\begin{array}{l}\text { Rating tool } \\
\text { (examples shared in supplementary files) }\end{array}$ & Rating tool & Rating tool \\
\hline & & & Standardized patient script \\
\hline \multirow[t]{3}{*}{ Materials } & $\begin{array}{l}\text { Printed material (case scenario, cue cards } \\
\text { with findings and age of patient) }\end{array}$ & $\begin{array}{l}\text { Printed material (description } \\
\text { of scenario, cue cards with } \\
\text { findings and age of patient, } \\
\text { drug calculation cards) }\end{array}$ & Printed material (case scenario) \\
\hline & Mannequin & Mannequin & \\
\hline & $\begin{array}{l}\text { Clinical supplies required to complete } \\
\text { task }\end{array}$ & $\begin{array}{l}\text { Clinical supplies required to } \\
\text { complete task }\end{array}$ & $\begin{array}{l}\text { Supplies required to complete } \\
\text { task }\end{array}$ \\
\hline
\end{tabular}

Note. The focus, personnel, documents and materials used are outlined for each of the 3 case stations included in the OSCE. 
Table 3. Time points of OSCE within PNEP

\begin{tabular}{|l|l|l|}
\hline \multirow{2}{*}{ Time point } & OSCE purpose & \multicolumn{2}{|l|}{ For program evaluation } \\
\cline { 2 - 3 } & For student assessment & Baseline measures of competency \\
\hline Prior to the program & & \\
\hline Throughout the program & $\begin{array}{l}\text { Formative assessment for student learning and } \\
\text { competency assessment prior to clinical practicum }\end{array}$ \\
\hline At graduation & Summative assessment for certification as pediatric RNs & Effectiveness of the program \\
\hline One-year post-graduation & & $\begin{array}{l}\text { Measures of maintaining competency } \\
\text { over time }\end{array}$ \\
\hline
\end{tabular}

Note. Summary of the time points of OSCE conduction within PNEP and the purpose of each OSCE for either student assessment or program valuation.

\subsection{Pilot testing the OSCE}

After developing the cases and rating tools, a pilot test was undertaken with faculty to determine feasibility and identify areas for improvement. To test the quality of the rater training developed, 2 raters were assigned to each OSCE station. Inter-rater reliability was calculated by the evaluation team to determine the degree of agreement between the raters (data not shown). Along with debriefs, this demonstrated that the training was effective, and led to the decision to assign 1 rater to each station in subsequent OSCEs. Debrief sessions with test raters also revealed that the initial 3-point rating scale was not sufficiently sensitive, leading to the development of a 5-point scale (see Figure 1).

\subsection{Rating scales}

To ensure that rating scales were appropriate for the Ghanaian setting, context-relevant terminology and concepts were developed through stakeholder consultation. A 5-point scale, consistent with a previously validated Bondy scale commonly used in OSCEs, was adapted to assess the students' standard of procedure; quality of performance; and level of assistance required to complete the skill ${ }^{[20,21]}$ (see Table 4).
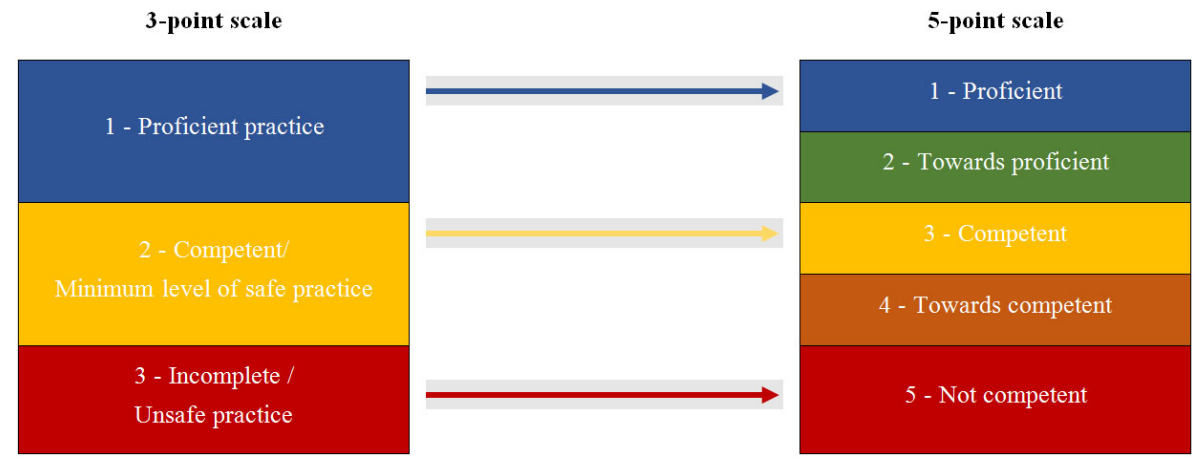

Figure 1. Adaptations made to Bondy scale during pilot testing

A rating scale was also developed for SPs to evaluate their satisfaction with student performance on the communication station. This employed a five-point scale, ranging from poor (1) to excellent (5), and served as a proxy for patient satisfaction.

\subsection{Rater and SP training}

Raters were selected based on their clinical background, availability, and level of familiarity with the foundational concepts of PNEP. Training took place prior to each OSCE, regardless of experience. Initial training took 2 days, and refresher training included 2 half-day sessions prior to each subsequent OSCE. There were 3 core components: review of the clinical competencies and practical skills being assessed; introduction to the cases and rating tool; and peer-to-peer practical sessions. Within the practical session, raters practiced using the rating tools while other faculty simulated the role of the student. The practical sessions also allowed raters to further discuss the rating of the OSCEs to ensure consistency.

For the communication skills station, which incorporated the use of SPs to portray the parent of a sick child, scripts were developed. The scripts included key messages that the SP was to relay to students during the exam, including suggested dialogue specific to students' actions. Training included review of the case and script; watching a video of a similar case; and a practical session with the raters. 
Table 4. Five-point adapted Bondy scale used for OSCEs in PNEP

\begin{tabular}{|l|l|l|}
\hline Rating & Classification & Characteristics \\
\hline ND & Not done & Behaviour/procedure not completed \\
\hline 1 & Not competent & $\begin{array}{l}\text { Unsafe behaviour/procedure } \\
\text { Unskilled } \\
\text { Required continuous cues* }\end{array}$ \\
\hline 2 & Towards competent & $\begin{array}{l}\text { Requires guidance to ensure safety / not completely accurate } \\
\text { Procedure does not accomplish intended outcome } \\
\text { Unskilled, inefficient, excess time and energy } \\
\text { Required frequent cues* }\end{array}$ \\
\hline 3 & Competent & $\begin{array}{l}\text { Safe, accurate, intended outcome mostly achieved } \\
\text { Skilled, hesitant, some excess time or energy noted } \\
\text { Required occasional supportive comments** }\end{array}$ \\
\hline 4 & $\begin{array}{l}\text { Safe, accurate, achieved intended outcome } \\
\text { Proficient, minimal hesitation good pace } \\
\text { Requires minimal supportive comments** }\end{array}$ \\
\hline 5 & $\begin{array}{l}\text { Safe, accurate, achieved intended outcome } \\
\text { Proficient, confident, efficient speed and style } \\
\text { No supporting comments required }\end{array}$ \\
\hline $\begin{array}{l}\text { * Cues involved providing the student with directions or prompts to guide the student's completion of the behaviour or procedure } \\
\text { ** Supportive comments involved statements of encouragement or agreement with the student's behaviour or completion of the procedure. }\end{array}$ \\
$\begin{array}{l}\text { Description: Five-point Bondy scale adapted for assessing OSCE performance in the Ghanaian context, including the characteristics associated with } \\
\text { each rating }\end{array}$ & \begin{tabular}{l} 
Proficient \\
\hline
\end{tabular}
\end{tabular}

\subsection{Student preparation and examination roll-out}

As the OSCE was a novel evaluation tool in this setting, email and verbal communication templates were developed to guide messaging to students and ensure consistency. Prior to each exam, structured discussions were also facilitated by the Lead Coordinator to address students' concerns. The OSCE was run by an examination team over 2 days (Table 1). To reduce potential bias, raters were assigned to 1 of the 3 training sites where they had not worked before. To ensure confidentiality, students waited in an isolated and supervised area until their assessment. The station setup ensured privacy and decreased distractions. To facilitate consistency, technical experts in evaluation methods from SickKids and GCNM provided continued mentorship to the raters.

\subsection{Post-OSCE debriefing sessions}

Debrief sessions were held with raters on each day of the OSCE, and with students after the completion of each OSCE. These were open discussions and important to capturing what did and did not work well to improve the overall experience and provided a chance for students to reflect on their performance and identify areas for improvement.

\section{LESSONS LEARNED}

Overall, the OSCEs were run 14 times throughout the PNEP program. There were several lessons learned within the OSCE development, planning, implementation, and debriefing sessions that led to their successful usage for certification within PNEP.

Published by Sciedu Press

\subsection{Efforts around stakeholders and engagement}

With the transition from traditional practical exams to the use of OSCEs, advocacy to national oversight bodies was required to ensure its acceptance as a method of evaluation. Furthermore, the use of SPs had a positive, unintended benefit of community engagement, and engagement and support were also necessary for students to value and be comfortable with the OSCE process.

\subsubsection{Stakeholder engagement}

Initially, some Ghanaian stakeholders, such as the NMC, challenged the validity of a simulated environment as a rigorous student evaluation method compared with practical exams in the clinical setting. Because the NMC is responsible for ensuring standardized practice of nurses and midwives, and issuing certification for specialties such as pediatrics, their engagement throughout the OSCE development process was critical to ensuring support and success. A representative of NMC sits on the GCNM governing council and was engaged for program approvals. Endorsement was further generated by inviting them to observe and provide oversight during the first cohort's OSCEs.

\subsubsection{Using standardized patient to engage the community}

Eleven community members have acted as SPs during the OSCEs. Engaging SPs is a way for community members to contribute to the improvement of healthcare delivery by ensuring health workers are adequately prepared to deliver high quality patient and family-centered care. The SPs reported that their role changed their perception of nurses in Ghana 
and gave them a better appreciation of nurses' knowledge and skills.

\subsubsection{Student preparation to ensure value}

Students had differing exposure to the OSCE assessment format, so it was important to ensure that the OSCEs were a positive learning experience and that the results obtained were reflective of the competencies being assessed, as opposed to format familiarity. To overcome the format novelty, different types of student preparation were incorporated at each time point. For students with no previous experience, this included pre-program OSCE guidance and demonstrations of how to take an OSCE (e.g. how to interact with the mannequin or SP and 'talk aloud' so the rater could assess their knowledge). Once students had completed formative OSCEs throughout the program, less preparation was required, although they did experience increased anxiety and stress related to their need to pass for certification. Refresher sessions and additional time in skills lab were developed to address this.

\subsection{Feedback mechanisms for developing and testing tools}

Engaging in open and ongoing discussion between the PNEP partners, ensuring collaborative station development, and conducting pilot testing of the case scenarios and OSCE process prior to the formal implementation of the OSCEs offered an important chance to refine the characteristics, resources, and the evaluation of the cases, and ensured that they were content- and context-relevant.

\subsubsection{Testing case scenarios}

Faculty member feedback was a valuable component of finalizing the case scenarios for the bank of cases. It allowed for the identification of any components that were unclear and ensured the case could be completed in the allotted time. Using a communication case as an example, it was initially noted that the parent presented too many problems to the student, which took too long. Correspondingly, changes were made to reduce the complexity of this case and ensured it could be conducted within the time limit.

\subsubsection{Resources for OSCE development}

Developing a realistic, simulated environment is important for the OSCEs; however, the setup can be limited by available resources and require accommodations. The use of mannequins was an important part of ensuring the students could demonstrate clinical skills during the physical assessment and emergency management stations, but mannequins corresponding to differently aged children were not available. To overcome this challenge, without compromising the integrity of a case, clear communication to the students about the age of the respective mannequins was incorporated and successfully conveyed in the exam using cue cards.

\subsubsection{Refining the rating scale}

As noted, the 3-point scale used during the pilot OSCE was not sensitive enough to identify between students with borderline performance and those who exceeded expectations (i.e. the difference between competent and proficient), leading to the majority of the students being labelled as proficient. With the revision to the 5-point adapted Bondy scale, detecting variability became possible and the local collaborators described it as a "more fair way to assess and differentiate students' performance". Further adding to its contextual relevance, the 5-point scale more closely aligned with common rating scales used throughout Ghana.

\subsection{Approaches to limiting rater bias}

Because a strength of the OSCE is standardization of the student examination experience, methods to limit potential rater bias were key, particularly for rater training and minimizing rater-student familiarity.

\subsubsection{Training for raters}

Inter-rater reliability measures functioned to assess the quality of training during the pilot OSCE. Lead OSCE trainers were identified in this process, which strengthened the ability to provide consistent and quality training. Feedback from the raters demonstrated that they felt prepared and confident to complete the student assessments.

\subsubsection{Strategies to minimize bias}

To minimize rater bias, raters were rotated between the training sites so that they would assess students unknown to them. Although travel was an additional expense, students described feeling that they were tested fairly when assessed by an unknown rater.

\subsection{Process integrity}

\subsubsection{OSCE protocols}

The OSCE protocols were important to ensuring consistent processes were followed between sites and over time. When challenges arose, they offered important guidance as to how to respond accordingly.

\subsubsection{Academic integrity}

Maintaining academic integrity is an overarching priority at GCNM, given its centrality to learning, teaching, and practice. This was upheld by ensuring the confidentiality of the cases and tools, as well as carefully managing materials and protocols for students, raters, and SPs to prevent information sharing. It was also integral to maintain a fair and consistent examination experience. 


\section{Discussion}

We developed the first OSCE for use in the Ghanaian context to assess clinical skill proficiency in pediatric nursing. While nursing students in this setting have traditionally been evaluated using practical exams, the collaborative development of this setting-specific OSCE, tailored to prioritized clinical competencies, offered a more standardized approach and incorporated the assessment of the knowledge, skills, and attitudes required for competent practice. The OSCE was both approved by national bodies for certification and successfully implemented, supporting its applicability and utility in a resource-limited setting.

Although reservations were initially expressed around the utility of the OSCEs for student assessment, both students and faculty recognized their value for both learning and impartiality. A previous study by Brighton, et al. noted that students found OSCEs a stressful but effective method of evaluation. ${ }^{[22]}$ From debriefs conducted throughout PNEP, students concluded that even with the stressful context of the assessment, the OSCE offered a valuable learning experience and raters were supportive. Students found the interactions with SPs especially valuable, as SPs offered an opportunity to challenge their communication skills in a supportive environment even though it was a new experience, having not interacted with SPs previously. Kenny et. al have similarly described the benefit of SPs to nursing students for integrating realism within learning communication skills and the empowerment such engagement can offer. ${ }^{[5]}$ Future integration of SPs within formative and summative assessments and learning could further enhance their use in this setting.

Despite the demonstrated successes, the national use of OSCEs for student certification is uncertain beyond the project period. GCNM will continue to use OSCEs throughout the academic year for formative assessments to provide feedback to students on their progress in preparation for clinical practicum. However, future post-graduate pediatric program nursing students will likely undergo traditional practical exams in the clinical setting for certification. Stated concerns for sustaining OSCEs for certification include resource constraints and general preference for the traditional method of practical exams, as it is felt to better reflect the setting that students will practice in upon graduation. This highlights the ongoing challenges around buy-in and the potential burden of increased resources and time required in conducting the OSCE.

Because the COVID-19 pandemic overlapped with the completion of the final PNEP OSCEs in September 2020, we had to adapt the OSCE format to eliminate the movement of education staff and reduce the possibility of transmitting the virus between teaching sites. This meant that raters Published by Sciedu Press often assessed their own students. To minimize potential rater bias, additional advanced training was provided, which emphasized fairness and included strategies to reduce bias. Ultimately, this adaptation demonstrated the possibility of decreasing the cost of running the OSCEs without compromising the assessment, given the reduced cost for rater travel and lodging. This more cost-effective OSCE format could offer an opportunity for future use and should be further evaluated considering the undeniable benefit of standardized, fair assessments within nursing certification.

\section{Conclusion}

As a form of student assessment, OSCEs are an important part of health care education. Our development of contextspecific OSCE tools and cases, rigorous training, and efforts around standardization demonstrated that the OSCEs could be a valid and credible method of student assessment in a resource-limited setting. Ongoing advocacy and research to further demonstrate the value and cost-effectiveness will be required for continued uptake of the OSCE as the national standard in Ghana. The lessons shared are informative for training institutions in the sub-region and other low-resource settings considering adopting OSCEs for student assessment.

\section{FUNDING}

The SickKids-Ghana Pediatric Nursing Education Partnership was funded by Global Affairs Canada and the SickKids Foundation with in-kind contributions provided by the Ghana Ministry of Health. Global Affairs Canada and SickKids Foundation had no formal role in any PNEP activities and were provided with regular reporting. Ghana Ministry of Health officials had no direct formal role in OSCE activities but were engaged in various committees that oversaw PNEP operations and were also provided with results of the program through regular committee reporting.

\section{ACKNOWLEDGEMENTS}

The authors wish to acknowledge the Ghana Ministry of Health, Ghana Health Services, Ghana College of Nurses and Midwives, and The Hospital for Sick Children (SickKids) for their commitment to the health of children in Ghana. Special thanks to members of the project management team including Chishuvo Mandivenga, Faustina Okae, Cindy Fiore and Nik Agarwal for coordination and logistics support. Bonnie Fleming-Carroll and Hannah Acquah along with a dedicated education team at SickKids and GCNM contributed significant expertise for OSCE development. Thank you to Jo-Anna Baxter for providing editorial assistance.

\section{CONFlicts OF InTEREST Disclosure}

The authors declare that there is no conflict of interest. 


\section{REFERENCES}

[1] Arlington L, Kairuki AK, Isangula KG, et al. Implementation of "Helping Babies Breathe": A Three-Year Experience in Tanzania. Pediatrics. 2017; 139(5): 1-10. PMid:28557724 https ://doi.or g/10.1542/peds. 2016-2132

[2] Elliott S, Pellowe K, Murrell P, et al. A comprehensive systematic review of the use of simulation in the continuing education and training of qualified medical, nursing and midwifery staff. JBI Library of Systematic Reviews. 2011; 9(17): 538-87. PMid:27819938 https://doi.org/10.11124/jbisrir-2011-135

[3] Williams BW, Byrne PD, Welindt D, et al. Miller's Pyramid and Core Competency Assessment: A Study in Relationship Construct Validity. J Contin Educ Health Prof. 2016; 36(4): 295-9. PMid:28350312 https://doi.org/10.1097/CEH.0000000000000117

[4] Lopreiato JO, Sawyer T. Simulation-based medical education in pediatrics. Academic Pediatrics. 2015; 15(2): 134-142. PMid:25748973 https://doi.org/10.1016/j.acap.2014.10.010

[5] Kenny G, Cargil J, Hamilton C, et al. Improving and validating children's nurses communication skills with standardized patients in end of life care. Journal of Child Health Care. 2016; 145-52. PMid:25395598 https ://doi.org/10.1177/13674935145555 88

[6] Gormley G. Summative OSCEs in undergraduate medical education. The Ulster Medical Journal. 2011; 80(3): 127.

[7] Traynor M, Galanouli D. Have OSCEs come of age in nursing education? British Journal of Nursing. 2015; 24(7): 38891. PMid:25849236 https://doi.org/10.12968/bjon. 2015 .24 .7 .388

[8] Dixon-Woods M, Lame G. Using clinical simulation to study how to improve quality and safety in healthcare. 2018.

[9] Pell G, Homer MS, Roberts TE. Assessor training: its effects on criterion-based assessment in a medical context. International Journal of Research \& Method in Education. 2008; 31(2): 143-54. https://doi .org/10.1080/17437270802124525

[10] Chong L, Taylor S, Haywood M, et al. The sights and insights of examiners in objective structured clinical examinations. J Educ Eval Health Prof. 2017; 14(34): 1-8. PMid:29278906 https://doi .or g/10.3352/jeehp. 2017.14.34

[11] Stroud L, Herold J, Tomlinson G, et al. Who You Know or What You Know? Effect of Examiner Familiarity With Residents on OSCE
Scores. Academic Medicine. 2011; 86(10): S8-S11. PMid:21955775 https ://doi.org/10.1097/ACM.0b013e31822a729d

[12] Holmboe E, Hawkins R. Practical Guide to the Evaluation of Clinical Competence. United States of America: Elsevier; 2008.

[13] Ameh CA, Kerr R, Madaj B, et al. Knowledge and Skills of Healthcare Providers in Sub-Saharan Africa and Asia before and after Competency-Based Training in Emergency Obstetric and Newborn Care. PLOS One. 2016; 1-14. PMid:28005984 https ://doi.org/ 10.1371/journal pone. 0167270

[14] Munkhondya TEM, Msiska G, Chilemba E, et al. Experience of Conducting Objective Structured Clinical Evaluation (OSCE) in Malawi. Open Journal of Nursing. 2014; 4: 705-13. https ://doi .org/10 .4236 /ojn. 2014.410075

[15] Katowa-Mukwato Pea. Implementation of Objective Structured Clinical Examination for Assessing Nursing Students' Clinical Competencies: Lessons and Implications. Creative Education. 2013; 48-53. https://doi.org/10.4236/ce.2013.410A008

[16] Onwudiegwu U. OSCE: Design, Development and Deploymen. Journal of the West African College of Surgeons. 2018; 1-22.

[17] Salehi R, Asamoah A, de Young S, et al. Scaling up pediatric nurse specialist education in Ghana- a longitudinal, mixed methods evaluation. BNC Nursing. 2021. PMid:33593320 https ://doi .org/10 .1186/s12912-021-00550-1

[18] Kelly MA, Mitchell ML, Henderson A, et al. OSCE best practice guidelines - applicability for nursing simulations. Advances in Simulation. 2016; 1(1): 1-10. PMid:29449979 https : //doi .org/10.1 186/s41077-016-0014-1

[19] Kachur E, Zabar S, Hanley K, et al. Organizing OSCEs (and other SP exercises) in ten steps. 2013; 7-34. https ://doi .org/10.100 7/9781461437499_2

[20] Hawly R, Lee J. Standardised clinical evaluation using Bondy rating scale. The Australian journal of advanced nursing: a quarterly publication of the Royal Australian Nursing Federation. 1991; 8(3): 6-10.

[21] Bondy KN. Criterion-referenced definitions for rating scales in clinical evaluation. Journal of Nursing Education. 1983; 22(9): 376-82. https : //doi .org/10.3928/0148-4834-19831101-04

[22] Brighton R, Mackay M, Brown RA, et al. Introduction of undergraduate nursing students to an objective structured clinical examination. Journal of Nursing education. 2017; 56(4): 231-4. PMid:28383748 https ://doi.org/10.3928/01484834-20170323-08 